
cultures

Les cahiers de l'Acedle

\title{
L'action en classe de langues et les activités de recherche en didactique des langues et des cultures
}

Réponse à Dominique Macaire

\section{Georges Daniel Véronique}

\section{(QpenEdition}

\section{Journals}

Édition électronique

URL : http://journals.openedition.org/rdlc/5097

DOI : $10.4000 /$ rdlc.5097

ISSN : 1958-5772

Éditeur

ACEDLE

\section{Référence électronique}

Georges Daniel Véronique, «L'action en classe de langues et les activités de recherche en didactique des langues et des cultures ", Recherches en didactique des langues et des cultures [En ligne], 4 | 2007, mis en ligne le 16 juin 2007, consulté le 11 septembre 2019. URL : http://journals.openedition.org/ rdlc/5097 ; DOl : 10.4000/rdlc.5097

Ce document a été généré automatiquement le 11 septembre 2019

\section{(c) (i) (9)}

Recherches en didactique des langues et des cultures is licensed under a Creative Commons AttributionNonCommercial-NoDerivatives 4.0 International License 


\title{
L'action en classe de langues et les activités de recherche en didactique des langues et des cultures
}

Réponse à Dominique Macaire

\author{
Georges Daniel Véronique
}

1 Le propos de cette contribution, qui s'adosse à une pratique d'enseignement en français, langue seconde et étrangère et à des activités de recherche dans le domaine de l'acquisition du français, est de proposer quelques réflexions à propos des objets et des méthodes de recherche en didactique des langues et des cultures (désormais DLC). Tout d'abord, je voudrais analyser brièvement, dans une première partie, la position de la DLC en tant que "discipline d'intervention». Je souhaite m'interroger, ensuite, réagir à l'intervention de Dominique Macaire sur la recherche-action. Je partage largement ses propos ; mes observations seront donc convergentes. Pour conclure la deuxième partie de cette contribution, j'évoquerai l'émergence d'une discipline au carrefour des recherches sur l'acquisition des langues étrangères et de la DLC, discipline qui illustre divers enjeux méthodologiques.

\section{La didactique des langues et des cultures, une discipline d'intervention}

2 La DLC est une discipline ancienne qu'un rapport de constitution unit aux disciplines de réflexion sur les langues et le langage. Orientée différemment de ces démarches théoriques mais en complémentarité et en rapport de nécessité mutuelle avec elles, la DLC a pour finalité de réfléchir aux conditions et aux moyens de la transmission des pratiques langagières et culturelles en milieu institutionnel. Il s'agit donc d'une discipline praxéologique, qui est en dialogue avec les sciences de l'éducation tout autant qu'avec les sciences du langage. 


\subsection{Un rapport de constitution à la grammaire et à la théorie du langage}

3 La didactique des langues et des cultures (DLC), en tant que discipline de l'action éducative linguistique, est associée de longue date à des pratiques d'enseignement, bien décrites par les historiens (Becchi \& Julia éds. 1998) ; cette discipline est évoquée dans de nombreux traités de pédagogie depuis l'Antiquité (Garin 1998), même si l'appellation DLC n'y a pas cours. L'art d'enseigner les langues, en rapport avec la tekhné grammatiké, ne reçoit la désignation de "didactique" qu'au dix-septième siècle (Besse 1998); son existence ancienne est cependant intimement liée à celles de la grammaire et de la philologie. Poser un rapport de continuité entre le grammaticus, le grammairien, et le chercheur-didacticien, considérer qu'un rapport de constitution unit la didactique des langues à la grammaire et, ultérieurement à la linguistique, c'est reconnaître le lien bi millénaire qui s'est tissé entre les réflexions sur les langues et le langage et les travaux en vue de leur transmission.

4 Pour mieux appréhender les enjeux de cette relation de constitution entre la DLC et une théorie du langage au sens étendu que lui donne Meschonnic, englobant non seulement les sciences du langage et la philologie mais également la poétique (Meschonnic 1991, voir Beacco \& Véronique 2005), l'on peut se pencher sur la notion clé de «langue-culture » et sur son utilité en DLC. Le terme de « langue-culture » fait son apparition en didactique / didactologie des langues dans les années quatre-vingts, sans doute en opposition à celui de «langue», usité chez les linguistes. Galisson 1986 l'emploie, associé au terme de lexiculture, pour désigner une orientation nouvelle de son activité lexicographique. Selon Galisson 1997: 80, «(...) les langues-cultures ne sont pas des disciplines scolaires spécialement propres à cultiver la rationalité (...) Par contre, les langues-cultures sont riches de potentialités pour la mobilisation et le perfectionnement de la sensibilité ». Pour cet auteur, la «langue-culture » constitue l'une des huit catégories éducatives fondamentales; les autres sont le sujet, l'agent, le groupe, le milieu institué, le milieu instituant, l'espace et le temps.

5 Pour Galisson 1998: 110, dans «langues-cultures» «(...) le trait d'union a valeur épistémologique et symbolique, puisqu'il marque la consubstantialité de la «langue » et de la «culture», mais aussi et surtout l'égalité d'importance et de statut des deux constituants de l'objet d'étude ». À propos de la pragmatique lexiculturelle, Puren et Galisson, 1999 posent que « la spécificité de cette discipline est de proposer une entrée dans la culture par les mots, d'intégrer culture et langue dans une approche qui ne les disjoigne pas, qui respecte également leur intime consubstantialité, de privilégier la culture courante (donc la culture-action) sur la culture savante et d'accorder une grande importance à une culture lexicaliste de l'interculturel (...).»

La notion de langue-culture en didactique des langues ainsi définie permet de rendre compte de la diversité des pratiques linguistiques et langagières transmises dans les classes de langues. Elle assigne des objectifs et un programme à cette discipline. On en comprend l'intérêt quand on se penche, par exemple, sur la notion de 'langue seconde'. On s'accorde, en effet, à distinguer le français, langue seconde, du français, langue maternelle ou première, et du français, langue étrangère, sur la base des critères suivants : le degré de compétence de l'apprenant dans la matière enseignée (est-elle la langue de première socialisation ou une langue à statut différent, une langue privilégiée ? 
) et l'étendue du partage culturel avec la communauté linguistique cible (les apprenants et les situations d'enseignement qui relèvent de la didactique du français langue seconde sont réputés partager des valeurs, des normes, des aspirations, véhiculées par la langue cible, avec les francophones natifs) (voir, par exemple, Cuq 1991 : 138-139 et Dumont, B. et al. 2000 : 7). C'est bien en tant que langue-culture que le français, langue seconde se différencie du français, langue étrangère.

7 La notion de langue-culture pose d'emblée qu'il convient de ne pas dissocier une didactique de la culture de la didactique de la langue (Zarate 1986) et de réserver à l'enseignement de la culture-civilisation (Beacco 2000) une place centrale par rapport à la question de l'enseignement linguistique et communicationnel.

Cette définition de la notion de langue-culture inscrit la DLC dans une perspective actionnelle. Développer une telle perspective ne conduit pas obligatoirement à négliger les composantes linguistiques ni à embrasser la pragmatique telle qu'elle est définie, entre autres, par les théoriciens des actes de langage. Il s'agit plutôt de substituer à la notion de locuteur, idéal ou non, celui d'acteur social, à la notion de communauté linguistique, celui de formation sociale, et à la notion de langue celle de pratiques langagières, régulées par des enjeux sociaux, dont ceux de la construction des significations sociales et de l'identité. L'invitation à repenser la question de la langue fait partie du programme d'une sociologie des langues (Bourdieu 1971) et de certaines démarches en sociolinguistique. À partir de ses préoccupations praxéologiques propres, la DLC est en mesure de poursuivre un cheminement analogue.

\subsection{Les enjeux sociaux de la DLC : l'exemple du curriculum}

9 Le rapport de constitution qui unit la DLC aux theories du langage n'exclut pas qu'elle se préoccupe de cultures éducatives et de savoir-faire pédagogiques. La DLC est ainsi conduite à entretenir des rapports avec les disciplines qui étudient le fait et l'idéal éducatifs par exemple. La relation que la DLC entretient, en ses activités curriculaires notamment, avec les politiques éducatives et linguistiques des formations sociales où elle est mobilisée, la distingue des recherches grammairiennes et linguistiques.

10 L'étude du procès d'enseignement-apprentissage des langues-cultures, cœur de la recherche en didactique des langues, suppose de revisiter les doctrines et les pratiques éducatives qui modèlent les institutions et la nature même des interactions entre les acteurs de l'instance éducative. Il est frappant de voir que, longtemps, la recherche sur l'enseignement des langues se soit satisfaite, par exemple, d'un inventaire des méthodologies d'enseignement sans se préoccuper des propositions éducatives formulées en amont, hors desquelles elles n'auraient pu voir le jour. Ainsi, est-il nécessaire de mettre en relation le mouvement éducatif qui se développe dans les pays germanophones autour de l'œuvre de Pestolazzi et le mouvement de réforme de l'enseignement des langues vivantes en Europe à la fin du siècle dernier pour mieux le comprendre. Il convient de même de rapporter le volet didactique de la francisation des provinces au programme d'études que détaille Rollin, et, un siècle et demi plus tard, aux propositions méthodologiques de Carré, elles-mêmes marquées par la tradition éducative allemande (L. Puren 2004).

11 La définition de la notion de curriculum permet également de réfléchir aux liens qui unissent éducation et DLC. Cette notion relative à la structuration des contenus à enseigner implique sans doute, comme Bernstein (repris dans Forquin éd. 1997) nous 
invite à le penser, une réflexion sur l'ordre social qu'elle génère. Comme le rappellent Charlot et coll. (1992 : 26), la notion de curriculum «[...] au-delà des "programmes ", renvoie aux parcours scolaires, aux situations d'apprentissage, aux finalités éducative elles-mêmes». Les didacticiens de langues proposent une lecture plus restreinte de curriculum, ramenée alors à l'ensemble des décisions à prendre dans l'organisation d'un contenu d'enseignement, en concurrence avec le syllabus, comme l'indique Lehmann (1993 : 144-149). L'article « curriculum » du Dictionnaire de didactique du français (Cuq 2003) met en relief les sens suivants :

- l'action de rationalisation conduite par des décideurs de l'éducation pour structurer un parcours d'apprentissage,

- le parcours éducationnel propre à l'apprenant.

Que l'on adhère à une vision restreinte ou élargie de la notion de curriculum, l'on touche ici à la relation complexe qui unit la définition d'un contenu à enseigner, des dispositifs d'action éducative et les enjeux sociaux de ces choix.

La DLC doit s'occuper de didactisation, ce qui implique certes un regard de linguistegrammairien mais pas seulement. En effet, c'est moins la langue des linguistesgrammairiens qui est au centre de ses activités que la socialisation dans une langueculture autre pour les apprenants. Discipline de l'action sociale, elle ne peut méconnaître les effets sociaux des dispositifs d'enseignements qu'elle prône. Ainsi entendu, l'on conçoit sans difficulté que la recherche-action soit envisagée comme son mode d'intervention privilégiée.

\subsection{Perspectives actionnelles}

Définir la DLC comme une discipline interventionniste, selon une formule de Galisson, place la conception et la réflexion sur les tâches et les dispositifs d'enseignement au cœur de ses travaux. C'est d'ailleurs à l'aune de l'activité d'enseignement qu'il convient de construire et d'évaluer la recherche en didactique. Cette perspective actionnelle implique que l'on se penche sur l'intentionnalité des actions d'enseignement, sur leur déroulement et sur l'évaluation de leurs résultats; compréhension et explication relèvent alors d'un même mouvement de recherche.

D'après H. Besse (1995), la DLC mobilise trois ensembles de discours : des discours réflexifs et d'élaboration de méthodologies, des discours de conception d'outils et de dispositifs d'enseignement et les discours de la classe. Dans cette contribution, il sera essentiellement question du discours de conceptualisation des activités didactiques.

L'intérêt de la perspective actionnelle en DLC est de remettre au centre de la réflexion didactique les acteurs sociaux que sont les enseignants et les apprenants et leur coactions. Comme le relève C. Puren 2006, l'agir communicationnel est dorénavant l'un des aspects de l'agir ensemble, non le type exclusif d'action à privilégier. Il s'agit donc de dégager les significations sociales co-construites à travers la médiation de l'enseignant et $d u$ matériau d'enseignement. On aurait tort cependant de ne pas entendre la mise en garde de Chiss (2005) qui invite à penser non seulement «[...] ce que l'on fait avec le langage mais à ce que le langage fait au sujet et au monde ». J'y reviens infra. 


\section{De la méthodologie de la recherche en DLC}

17 En DLC, comme dans d'autres disciplines, les questions de base en matière de méthodologie de la recherche portent toujours sur l'identification des objets de recherche et sur les motifs de ces investigations, sur l'évaluation et la validation du processus de recherche et de ses résultats et sur les rapports entre l'activité enseignante et la recherche.

\subsection{L'intervention de Dominique Macaire sur la recherche-action en DLC}

18 À la suite de Narcy-Combes 2005, Dominique Macaire (dorénavant D.M) prône la recherche-action comme une des modalités principale de recherche en didactique, elle s'interroge cependant i) sur les rôles et les statuts des chercheurs et des praticiens dans une telle entreprise ii) sur la double posture du chercheur entre implication et observation et iii) sur la duplication de telles recherches. Je partage ses interrogations; j'y apporterai des réponses un peu différentes.

19 Sur la base d'une riche expérience, D.M. pose non seulement que la recherche-action est la forme d'investigation qui s'impose en DLC mais prône un positionnement ethnographique dans la conduite du travail sur le terrain, une démarche herméneutique attentive aux accidents et aux difficultés du terrain. Elle pose que ce mode d'inscription devrait non seulement permettre au processus de recherche de se dérouler mais permettre d'y engager les différents acteurs. On peut, certes, imaginer des dispositifs de recherche-action où les enseignants sont également des chercheurs. Ce type d'organisation de la recherche, prônée par Nunan (1991) et Crookes (1997) par exemple, est certainement bénéfique pour l'enseignant et pour l'innovation pédagogique. Elle soulève des questions quant à sa mise en forme, à la diffusion des activités de recherche qu'elle génère, et quant à la validation de la recherche, au-delà de l'action effective en classe. Le rôle et le statut des chercheurs et des praticiens, selon la formule de D.M, me semblent définis non seulement par le mode d'investigation retenue mais également par son aboutissement, la communication des résultats de la recherche. Le monde de la "science en action" présente des aspects "nocturnes", clairement identifiés par Bourdieu (2001), qui laisse à penser que chercheurs et praticiens courent inégalement le risque de la légitimité de parole.

Le praticien, en tant que tel, obéit à des impératifs qui sont ceux de l'activité qu'il conduit. Il lui est difficile de fait, même aidé par le chercheur, de participer d'une autre sphère d'activité, dont par principe les enjeux ne sont pas les siens. Le risque est grand de se sentir trahi dans ce nouveau domaine d'activité.

Pour ma part, tout en étant favorable au dispositif de la recherche-action, qui permet de réinvestir dans le temps de la recherche, ses effets, voire ses résultats, je demeure attaché à l'examen des possibilités de triangulation de la recherche, à défaut de pouvoir la réitérer. En effet, il faut que des sources indépendantes puissent confirmer ce que l'on aurait tendance à attribuer à l'effet de la recherche-action, sans pouvoir le vérifier. Il convient de se prémunir de l'effet pygmalion qu'introduit toute innovation dans un 
univers institutionnel, qu'elle bouscule par le fait même de sa structuration. Il faut également se prémunir du risque d'être juge et partie.

\subsection{Une réflexion à partir de la relation entre les recherches sur l'acquisition des langues et la DLC}

Je souhaiterais, pour conclure, évoquer le cas de l'émergence d'une discipline de recherche à l'intersection des recherches sur l'acquisition des langues (RAL) et de la DLC, en France et ailleurs dans le monde. Cette branche de la recherche en DLC résume, à elle seule, quelques-unes des difficultés de la posture de recherche dans le domaine praxéologique. La difficulté de la mise en dialogue des recherches linguistiques sur l'appropriation linguistique et des travaux en DLC tient aux différences d'orientation et de finalité des disciplines en question, à des thématiques de recherche divergentes, à des effets de doxa indissociables des activités auxquelles se livrent didacticiens et linguistes (voir par exemple les 'philosophies spontanées' qui animent les uns et les autres à propos de l'activité enseignante ou à propos de la langue) et à des différences de mode de démonstration et d'argumentation. Il s'agit en effet de concilier une observation attentive de l'appropriation linguistique par les élèves et des impératifs de didactisation et d'organisation des enseignements.

Dès les années 80 , se met en place, dans les pays de langue anglaise, une second language classroom research (recherche sur les classes de langues étrangères) (Nunan, 1991), qui se différencie dans ses orientations des travaux de recherches linguistiques sur l'acquisition des langues. À la même période, la Sprachlehrforschung (recherche sur l'enseignement des langues) voit le jour dans les pays de langue allemande (Edmondson \& House, 2000). Pour Nunan 1991, la second-language classroom-oriented research présente au moins l'un des deux traits définitoires suivants: les données ont été recueillies en salle de classe et la recherche tente d'apporter une réponse à des questions de pédagogie des langues. Il s'agit soit de produire des travaux sur l'apprentissage en classe qui dépassent la dichotomie du qualitatif et du quantitatif, soit de prendre en compte du point de vue de l'enseignement des langues des résultats de la RAL.

Depuis au moins 1987 (Blanc et al., éds., 1987), le domaine francophone a exploré régulièrement les pratiques langagières en classe de langue. Berthoud \& Py (1993), dans un ouvrage en direction des enseignants de langues, présentent, entre autres, “ l'interaction verbale comme lieu, moyen et objet d'acquisition ". Parmi quelques recherches substantielles en ce domaine, on citera celle de Clerc (1997), consacrée à l'acquisition de compétences narratives en milieu institutionnel, celle de Pekarek (1999) qui lie étude des représentations et acquisition de pratiques discursives, les travaux réunis dans la livraison 12 d'AILE (Pekarek \& Mondada éd, 2000) ou dans Cicurel \& Véronique éd (2002). S'il existe bien de nombreux travaux sur l'apprentissageenseignement d'une L2 en classe, cela ne veut pas dire que l'on dispose véritablement de recherches sur l'acquisition en classe (voir, cependant, Carol, 1995 et Granget, 2004, par exemple).

En dépit d'une proposition de Bouchard (1992), un secteur de recherche comparable à ceux qui se sont affirmés en contexte germanophone ou anglophone, unissant les travaux des didacticiens et des acquisitionnistes en un nouvel ensemble, distinct de la RAL, n'a pas vu le jour en contexte francophone. Cela s'explique sans doute à la fois par les priorités de la DLC en secteur francophone où les travaux sur l'appropriation sont peu importants et 
par un absence d'intérêt symmétrique des linguistes pour l'apprentissage en classe de langues. Un tel domaine pourrait, pourtant, retenir le programme esquissé par M.T. Vasseur et J. Arditty (Arditty \&Vasseur, 1999, 2002), programme peu éloigné par certains aspects de la Sprachlehrforschung.

\section{Conclusion}

Discipline fondée sur des pratiques au moins bi millénaires, la didactique des langues et des cultures apparait, dans le contexte épistémologique contemporain, comme une discipline nouvelle. L'interdépendance des disciplines, les frontières mouvantes des savoirs, la nécessité où l'on se trouve de distinguer des sciences nomothétiques, comme la linguistique ou la psychologie, des technologies et des praxéologies, créent des proximités et des différenciations nouvelles. C'est ce que j'ai tenté de montrer à propos de la notion de «langue-culture » ou celle de "curriculum ». Le passage d'une linguistique appliquée à l'enseignement des langues à une didactique des langues dans les recherches de langue française dans les années soixante-dix, répond à des nécessités locales. Ce mouvement qui est à l'origine de la DLC des années quatre-vingts, a eu pour effet indirect d'empêcher qu'un partie des travaux sur l'appropriation des langues évoluent vers la second language classroom research ou la Sprachlehrforschung. La mise en dialogue continue de réflexions sur la transmission et l'appropriation des langues et de disciplines connexes, telle celle de la recherche sur l'acquisition des langues étrangères, constitue à n'en point douter un bel enjeu de recherche théorique et méthodologique.

\section{BIBLIOGRAPHIE}

Arditty, J. \& M.-T Vasseur 1999. Présentation. In J. Arditty \& M.-T Vasseur (éd.). Langages n 134 , 3-19.

Arditty, J. \& M.-T Vasseur 2002. Contextes, variabilités et activités d'appropriation des langues. In F. Cicurel, F. \& D. Véronique (éd.). Discours, action et appropriation des langues, 251-264. Paris, Presses de la Sorbonne Nouvelle.

Article curriculum in Cuq, J.-P., 2003). Dictionnaire de didactique du français langue étrangère et seconde, Paris, CLE International, pp. 64-65.

Beacco, J.-C., Chiss, J.-L., Cicurel, F., Véronique, D. (éds.) 2004. Les cultures éducatives et linguistiques dans l'enseignement des langues. Pars, PUF.

Beacco, J.-C., 2000. Les dimensions culturelles des enseignements de langue. Paris, Hachette.

Beacco, J.-C., Véronique, D. 2005. Conclusion. Cultures linguistiques, éducatives et didactiques : parcours d'une notion et perspectives de recherche, pp. 269-276.

Becchi, E., Julia, D. (éds.). 1998. Histoire de l'enfance en Occident. 1. De l'antiquité au XVII siècle. Paris, Seuil. 
Bernstein, B., 1997. À propos du curriculum. In Forquin, J.-Cl. (éd.), Les sociologues de l'éducation américains et britanniques. Présentation et choix de textes. Paris, Bruxelles, De Boeck, pp. 165-171.

Berthoud, A.-Cl. \& B. Py 1993. Des linguistes et des enseignants. Berne, Peter Lang.

Besse, H. 1995. Méthodes, méthodologie et pédagogie. Le Français dans le monde. Recherches et Applications, 96-108.

Besse, H. 1998. Contribution à l'histoire du mot didactique. In Billiez, J. (éd.). De la didactique des langues à la didactique du plurilinguisme. Hommage à Louise Dabène, pp. 17-30, Grenoble, CDLLIDILEM.

Blanc, H., M. Le Douaron.\& D. Véronique (éd.) 1987. S'approprier une langue étrangère. Paris, Didier Érudition.

Bouchard, R. 1992. Présentation. In R. Bouchard et al. (éd.), Acquisition et enseignement/ apprentissage des langues, 5-13. Grenoble, Lidilem.

Bourdieu, P. 1971. Ce que parler veut dire. Paris, Plon.

Bourdieu, P. 2001. Science de la science et réflexivité. Paris, Raisons d'agir Éditions.

Carol, R. 1995. L'acquisition de la temporalité en allemand langue seconde par des apprenants guidés.

Thèse de doctorat, Lyon II.

Charlot, B., Bautier, E., Rocheix, J.-. 1992. École et savoir dans les banlieues et ailleurs ... Paris, Armand Colin.

Clerc, S. 1997.Étude de l'acquisition des conduites narratives en français langue étrangère et propositions didactiques. Thèse de doctorat, U. de Provence (http://www.marges-linguistiques.com [lien valide à la date de publication])

Crookes, G. 1997. SLA and Language Pedagogy. A socioeducational Perspective. Studies in Second Language Acquisition, vol. 19, $\mathrm{n}^{\circ} 1,93-116$.

Cuq, J.-P. (éd.) 2003. Dictionnaire de didactique du français langue étrangère et seconde. Paris, CLE International.

Cuq, J.-P. 1991. Le français langue seconde. Origines d'une notion et implications didactiques. Paris, Hachette.

Dabène, L. 1994.Repères sociolionguistiques pour l'enseignement des langues. Paris, Hachette.

Edmondson, W. \& J. House 2000. Einführung in die Sprachlehrforschung. 2. Auflage.Tübingen \& Basel : A. Francke.

Forquin, J.-Cl., 1994, Curriculum, in Champy, Ph., Étévé, Chr., éds. Dictionnaire encyclopédique de l'éducation et de la formation, Paris, Nathan, pp. 218-222.

Galisson, R. 1991. De la culture à la langue par les mots. Paris, CLE International.

Galisson, R. 1997. Les concepts fondateurs de la didactologie sont-ils des passeurs de gué légitimes ? Études de linaguistique appliquée, $\mathrm{N}^{\circ} 105,73-92$.

Galisson, R. 1998. À la recherche de l'éthique dans les disciplines d'intervention. Études de linguistique appliquée, $\mathrm{N}^{\circ} 109$, pp. 83-127.

Galisson, R., André, J.-Cl. 1998. Dictionnaire de noms de marques courants. Essai de lexiculture ordinaire. Didier Érudition, Paris.

Galisson, R., Puren, Chr. 1999. La formation en questions. Paris, CLE International. 
Garin, E. 1998. L'image de l'enfant dans les traités de pédagogie du XVe siècle. In Becchi, E., Julia, D. (éds.). 1998. Histoire de l'enfance en Occident. 1. De l'antiquité au XVII siècle, pp. 247-270, Paris, Seuil.

Granget, C. 2004. L'acquisition par des apprenants germanophones de la référence au passé en français : un cas d'école. Thèse de doctorat, Paris III.

Lehmann, Denis, 1993, Objectifs spécifiques en langue étrangère. Les programmes en question. Paris, Hachette.

Meschonnic, H. 1991. Le langage comme défi. In Meschonnic, H. (éd.). Le langage comme défi. Les Cahiers de Paris VIII, pp. 9-15, Paris, Presses Universitaires de Vincennes.

Mondada, L. \& S. Pekarek-Doehler 2000. Interaction sociale et cognition située : quels modèles pour la recherche sur l'acquisition des langues? AILE $n^{\circ} 12,147-174$.

Narcy-Combes, J.-P. 2005. Didactique des langues et TIC : Vers une recherche-action responsable. Gap, Paris, Ophrys.

Nunan, D. 1991. Methods in Second Language Classroom-Oriented Research : A Critical Review. Studies in Second Language Acquisition, 13, 2, 249-274.

Pekarek, S. 1999. Leçons de conversation. Dynamiques de l'interaction et acquisition de compétences discursives en classe de langue étrangère. Éditions universitaires Fribourg, Suisse.

Puren, L. 2004. L'école française face à l'école alloglotte. Contribution à une étude des politiques linguistiques éducatives mises en œuvre à l'égard des minorités linguistiques scolarisées dans le système éducatif français du XIX ${ }^{e}$ siècle à nos jours. Thèse de doctorat, Paris III.

Puren C. 2006. De l'approche communicative à la perspective actionnelle. Le français dans le Monde, 347, pp. 37-40.

Zarate, G., 1986. Enseigner une culture étrangère. Paris, Hachette.

\section{RÉSUMÉS}

Dans cette contribution, une réflexion est menée sur la nature de la didactique des langues et des cultures, qui est définie comme une discipline praxéologique. Différentes dimensions de la recherche-action qui pourrait être la méthodologie de recherche la plus appropriée de ce secteur sont discutées. On se penche, enfin, sur une branche de la didactique des langues et des cultures qui tentent de relier les travaux linguistiques sur l'acquisition des langues étrangères et la didactisation en classe de langues.

This paper is devoted to a discussion of the nature of language and culture didactics. The methodology and implications of research action in the field of language teaching is examined. The issue of second language classroom research is analyzed in the French context.

\section{INDEX}

Mots-clés : didactique des langues et des cultures, recherche-action, recherche en classe de langue seconde

Keywords : language and culture didactics, research-action, second language classroom research Schlüsselwörter : sprachlehr-und-lernforschung 
AUTEUR

GEORGES DANIEL VÉRONIQUE

Université de Provence, France ; Laboratoire DILTEC, Université Paris 3 Sorbonne Nouvelle, France 